\title{
Article \\ Suppressing Diffraction-Related Intensity Losses in Transmissive Single-Crystal X-ray Optics
}

\author{
Nataliya Klimova ${ }^{1, *(\mathbb{D})}$, Irina Snigireva ${ }^{2}$, Anatoly Snigirev ${ }^{1}$ and Oleksandr Yefanov ${ }^{3}$ \\ 1 International Science and Research Center "Coherent X-ray Optics for Megascience Facilities", \\ Immanuel Kant Baltic Federal University, 236022 Kaliningrad, Russia; anatoly.snigirev@gmail.com \\ 2 European Synchrotron Radiation Facility (ESRF), BP 220, 38043 Grenoble, France; irina@esrf.fr \\ 3 Center for Free-Electron Laser Science CFEL, Deutsches Elektronen-Synchrotron DESY, Notkestr. 85, \\ 22607 Hamburg, Germany; oleksandr.yefanov@cfel.de \\ * Correspondence: klimovanb@gmail.com
}

Citation: Klimova, N.; Snigireva, I.; Snigirev, A.; Yefanov, O. Suppressing Diffraction-Related Intensity Losses in Transmissive Single-Crystal X-ray Optics. Crystals 2021, 11, 1561. https://doi.org/10.3390/ cryst 11121561

Academic Editor: Aidar T. Gubaidullin

Received: 22 October 2021

Accepted: 10 December 2021

Published: 14 December 2021

Publisher's Note: MDPI stays neutral with regard to jurisdictional claims in published maps and institutional affiliations.

Copyright: (c) 2021 by the authors. Licensee MDPI, Basel, Switzerland. This article is an open access article distributed under the terms and conditions of the Creative Commons Attribution (CC BY) license (https:// creativecommons.org/licenses/by/ $4.0 /)$.

\begin{abstract}
The highest-quality X-ray optics can be made of single-crystal materials such as silicon, germanium, or, even better, diamond. Unfortunately, such X-ray optics have one drawback: diffraction losses or the "glitch effect". This effect manifests itself as follows: at some energies of X-rays, the intensity of the transmitted beam drops due to the fact that some crystalline planes have satisfied the diffraction condition. Diffraction losses are usually observed in spectroscopic experiments when the energy of the $X$-rays changes in a certain range. However, this effect might also influence any experiment using $X$-rays, especially at higher energies. In this paper, we propose a method to overcome the glitch problem in transmissive optics. This is achieved using small rotations of the optical element. We describe the algorithm for "glitch-free" measurements in detail and the theory behind it.
\end{abstract}

Keywords: X-ray glitches; single-crystalline X-ray optics; diffraction losses

\section{Introduction}

X-ray beams generated by new sources, such as free-electron lasers (FELs) and fourthgeneration synchrotrons, have outstanding brightness and coherence. To deliver such a beam to the sample, the quality of the optics has to be very high. X-ray optics, such as monochromators, compound refractive lenses (CRLs) [1], attenuators, windows, beam intensity monitors, etc., are usually made of either single-crystal or polycrystalline materials. Single-crystal materials, such as silicon, germanium, or diamond, are of high quality, are robust, and are easily reproducible. However, such optics have one drawback: diffraction losses due to undesired Bragg/Laue scattering, which is usually termed the "glitch effect" [2]. This effect manifests itself as follows: at some energy of the incident X-rays, the transmitted (or diffracted, in the case of monochromators) beam intensity drops. This happens when the Bragg's law is satisfied and a part of the beam is parasitically diffracted in some direction. Therefore, the main beam (transmitted, or diffracted for monochromators) loses some intensity (the parasitically diffracted part). For thick optical elements (for example some CRLs made of silicon or diamond), this effect can lead to almost zero transmitted intensity.

Optics made of polycrystalline materials, such as CRLs made of aluminum or beryllium, some attenuators, etc., are usually not affected by the glitch effect because each grain inside of the polycrystal has a different orientation. However, such optics have another drawback: the grain boundaries scatter X-rays, which increases the background and, with time, the optical properties of such optics can decrease due to transformations inside the polycrystalline material. In addition, such optics are usually less reproducible and actually less robust. To overcome the problem of the low robustness of beryllium or aluminum, CRLs made of polycrystalline diamond were tested [3-5]. However, this still could not counter the low quality of the focused beam. Therefore, for new generations of X-ray 
sources, such as FELs or fourth-generation synchrotrons, polycrystalline optics could be a limiting factor for the beam quality. Hence, the best choice would be the optics made of the single-crystal material but with the possibility of avoiding glitches.

The glitch effect was mostly observed in spectroscopic measurements, when the energy of the beam, incident on a studied sample, was continuously changing. However, this effect is much more universal-glitches can be observed at fixed energies, especially for hard X-rays where the density of the glitches is higher [6,7]. Considering the fact that the glitches are very sensitive to the orientation of the crystal [7], measuring close to a glitch can lead to not only a lower intensity, but also make the beam intensity unstable.

In the past, the glitches were mainly observed in monochromators [8-11] due to the fact that these are the main optical element made of single-crystal material, which are present in most of the instruments. However, the glitches in monochromators are always generated in multiple-beam diffraction conditions: when several sets of crystal planes (or reflections in reciprocal space) satisfy the Bragg condition simultaneously. Indeed, the main reflection of the monochromator crystals (for example 111) is always excited; hence, the glitches appear when some other reflection is excited simultaneously. Usually, the main reflection of the monochromator crystals is rather strong $(111,220,113,004)$, so the penetration depth of the X-rays is rather low due to the small extinction length. Therefore, the reflections that are weaker than the main one cannot scatter a large portion of the intensity (for weaker reflections the extinction length is higher).

For transmissive optics, the situation is completely different: for most energies no reflections are excited, therefore, the transmitted beam is affected only by the absorption. When some set of planes satisfies Bragg's law, the scattered portion of the beam depends on the reflection strength and the thickness of the transmissive optical element. Therefore, the intensity drop, caused by the glitches, can easily lead to a transmitted beam of zero intensity. This is why the glitches in transmissive optics are more dangerous than in monochromators.

As mentioned earlier, X-ray optics made of single-crystal materials, especially CRLs, are becoming increasingly popular in new generations of X-ray sources due to the outstanding quality of such materials. Therefore, such optics have been developed over recent years and are being installed at some beamlines. The novelty of such optics explains the fact that there are not as many papers regarding the glitches in transmissive optics as there are regarding the glitches in monochromators. The origins of the glitches in both transmissive and reflective $\mathrm{X}$-ray optics are rather similar, but the theory is quite different. Hence, we will briefly overview the methods used to counter glitches in monochromators, as most of these methods can also be applied to transmissive optics.

As already mentioned, due to the glitches, the intensity of the beam incident on the studied sample drops at some energies. The easiest way to correct for the drops in the intensity is to measure the intensity before the measured sample (I0) and then normalize the signal measured after the sample (I1) by the I0. Unfortunately, this method often still leaves some artifacts in the measured signal [10], so some other methods are needed.

One of the ways to avoid glitches is to decrease the crystallinity of the optics-for example, to use bent crystals, such as bent monochromators [8]. However, this method is technically difficult to implement and can also decrease the quality of the beam.

Another way to minimize the number of glitches is to align the optical element, so that the X-ray beam is parallel to one of its main crystallographic directions [12]. However, this just minimizes the issue, but does not eliminate it. In addition, such manipulations with the optical element could be impossible due to the absence of some motors in the setup. Some beamlines provide a table of the measured "glitches" [13]. However, this could only work if there are no changes in the setup of the beamline.

One more approach is to use the beam's change in polarization, which is due to a process termed "multiple-beam dynamical effects". By measuring the signal using a 2D detector, one can derive the glitches' influence from the spatial distribution of the beam at the $2 \mathrm{D}$ detector $[14,15]$. However, this method is complicated and might not work for 
arbitrary samples and beamline configurations. In addition, it might be unusable for the transmissive optics.

Another way to fight glitches is just to smooth the measured spectrum (with a highpass filter) [16]. This method works satisfactorily because the glitches usually produce sharp drops in intensity and a high-pass filter can eliminate most of the glitches. The problem with this method is that the actual measured data can also be affected. Alternatively, the regions affected by the glitches can just be excluded from further analysis, but by doing so one can also lose some useful information.

In this work, we propose an approach for avoiding glitches in single-crystal X-ray optics by utilizing the high sensitivity of glitches to the crystal orientation. We have developed a method for finding the exact orientation of the optical element with respect to the incident beam (already described in $[6,7]$ ) and describe the way to determine the exact rotation axes of the optical element. This information can be used for calculating small rotations of the transmissive optical element in order to avoid glitches at certain energies. The proposed method would require changing the way the energy scan is performed, but it would allow the usage of the transmissive single-crystal X-ray optics without diffraction losses.

\section{Materials and Methods}

\subsection{Experiment and Data Processing}

The experiments were performed at Swiss-Norwegian Beamline (SNBL) BM31. This is a bending magnet source at the European Synchrotron Radiation Facility (ESRF), France. During the beamtime, several different types of diamond samples were measured: a diamond plate (supplied by Element Six Ltd., Didcot, UK) [17], a one-dimensional CRL that consisted of two sets of compound refractive lenses (machined by Micro Usinage Laser MUL, Grattentour, France) [18,19], a one-dimensional CRL that consisted of three sets of compound refractive lenses (manufactured by New Diamond Technology NDT, Saint Petersburg, Russia) [20], and a two-dimensional lens (grown at the Technological Institute for Superhard and Novel Carbon Materials, Troitsk, Russia) [21]. All samples were measured in so-called EXAFS mode when the energy of the incident beam was constantly changed. At the same time, the intensities of the incident (I0), and the transmitted through the sample (I1) beams, were measured using ionization chambers (see Figure 1). The step of the scan was limited by the resolution of the monochromator, which was $1 \mathrm{eV}$. The sample was mounted at a three-cradle goniometer so it could be rotated around any axis during the measurements. More details about the samples and the experiment can be found in $[22,23]$.

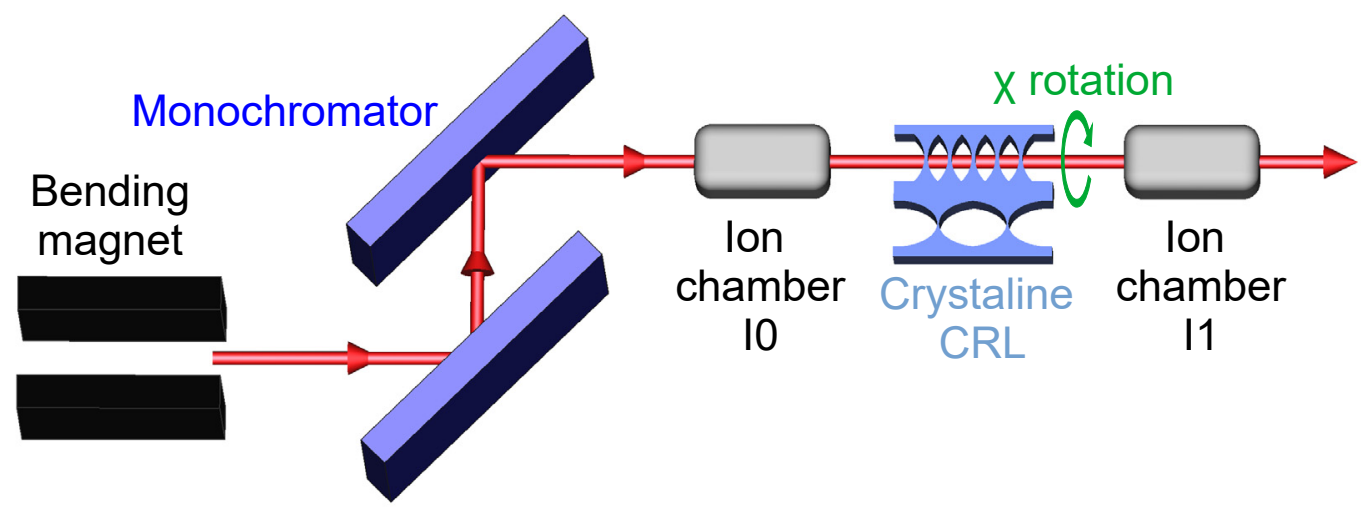

Figure 1. Glitch spectroscopy experiment at BM31 (SNBL), ESRF. The X-ray beam from the bending magnet is monochromatized and incident at a CRL. The intensity is measure before (I0) and after (I1) the sample with ion chambers.

For the first CRL (which we refer to as MUL) [19], we launched several scans at different angles $\chi$ (rotation around the beam-see Figure 1). The thick part of the lens (bulk) was scanned at $\chi=0^{\circ}, \chi=15^{\circ}$ and $\chi=30^{\circ}$. For most of the samples, the $X$-ray beam 
was parallel to the crystallographic direction [010]. Only for the second CRL (which we referred to as NDT), was the beam parallel to the direction [110].

The ion chamber I0 recorded the glitches generated by the monochromator (singlecrystal silicon) while the ion chamber I1 recorded the glitches caused by both the monochromator and the sample. Hence, to eliminate the glitches caused by the monochromator as well as to compensate for the refills of the synchrotron ring with electrons, the transmitted intensity was normalized by the incident intensity (I1/I0). The resulting spectra were also normalized by their smoothed (running median with a window size of 5 pixels) version to compensate for the changing transmission of the diamond for different energies and efficiencies in the beamline and ion chambers. An example of the normalized glitch spectra, together with the simulation, is presented in Figure 2.

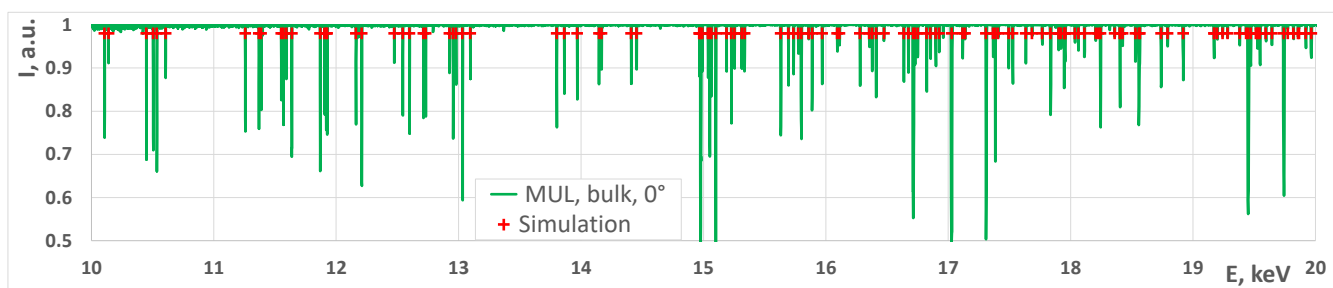

(a)

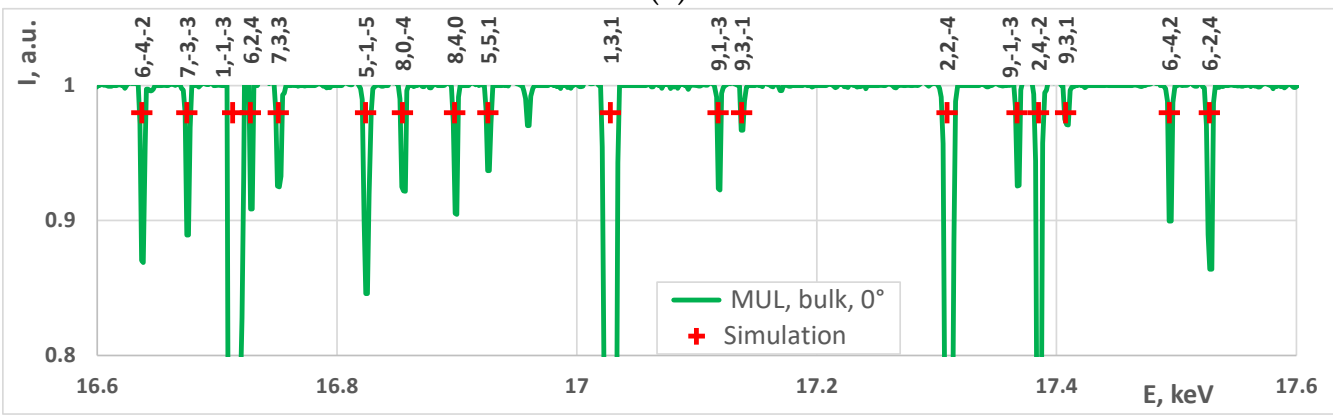

(b)

Figure 2. (a) Measured and simulated glitch spectrum for a diamond plate; (b) magnified part of the glitch spectrum for a diamond plate.

\subsection{Fitting the Glitches Spectra}

The exact energies of the glitches were found from the normalized spectra using the automatic procedure described in detail in $[7,24]$. After a list of glitches energies was generated, the fitting procedure was launched-the theory of glitches formation and the detailed description of the fitting procedure can be found in $[7,24]$. Also, the visual demonstration of the glitches formation is referenced from the Supplementary Materials. All programs written for this data processing can be found on GitHub [25] - see also the Supplementary Materials. An example of the fitted glitch spectra is presented in Figure 2.

During the data processing, we have found that the energy of the X-rays was recorded with some error due to the offset in the monochromator pitch angle (the details of this analysis are described in $[7,24])$. After correcting the energy (the correction was calculated from the data directly), the average square discrepancy (referred to as error) for all fitted spectra for all samples was in the order of $0.5 \mathrm{eV}$. Considering the step size of $1 \mathrm{eV}$ and the imperfect automatic determination of the exact glitch energy [7], the result can be considered to be good. Such a good result is also due to the fact that 100-120 glitches can be observed in diamond for the spectral range between 10 and $20 \mathrm{keV}$. Hence, this number of measurements has satisfactorily constrained the solution.

\subsection{Sensitivity of the Glitches to the Rotation of the Crystal}

During the analysis of the experimental data, we have found that the spectrum of glitches is very sensitive to the orientation of the optical element as well as to the cell 
parameters. Hence, a small change in the orientation of the lens leads to a considerable change in the glitch spectrum. An example of this effect is shown in Figure 3.

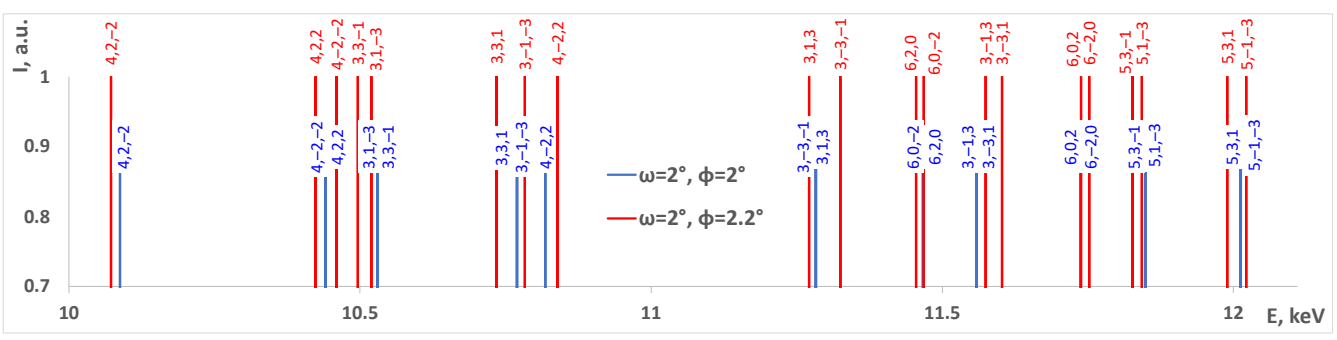

Figure 3. Simulated glitch spectra for MUL lens at $\omega=2^{\circ}$ and two $\phi$ angles: $2^{\circ}$ and $2.2^{\circ}$.

This fact can be used to avoid glitches at certain energies. As can be seen from Figure 3, most of the glitches shifted quite a lot after the lens was rotated by only $0.2^{\circ}$. That means that by performing small controllable rotations, we can shift the glitches from the energy we need to measure. However, to do this with a satisfactory precision, we need to accurately determine the rotation axis. Luckily, we can obtain this information from the glitch's spectra; we only need to measure two spectra at two different angles of the rotation stage that we plan to use for avoiding glitches.

\subsection{Avoiding Glitches by Tilting the Optical Element}

The possible glitches in diamond in the energy range of $10-16 \mathrm{keV}$ were simulated for different azimuthal angles $\phi$-Figure $4 \mathrm{a}$. Each line here represents a glitch corresponding to some reflection - see the legend. Hence, this figure represents the aforementioned dependence of the glitch energy on the orientation of the crystalline lattice. One can see that the number of glitches observed for any angle $\phi$ is usually almost constant, except for angles where the beam is parallel to some zone axis (for example, $\phi=0$ ). In the latter case, we can observe fewer glitches because many of the observed glitches correspond to several reflections, or reciprocal lattice points (RLPs), which are simultaneously excited. In addition, it is clear that for a fixed X-ray energy, changing the azimuthal angle leads to an intersection with some "glitch line" that results in the appearance of a glitch at that angular position.

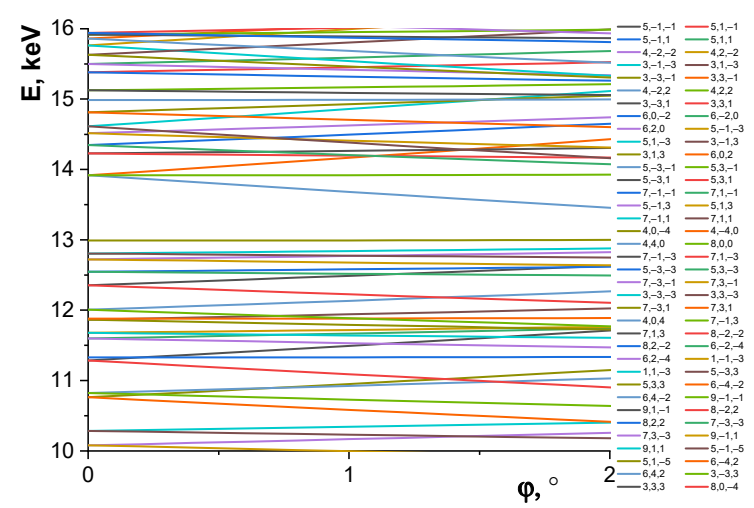

(a)

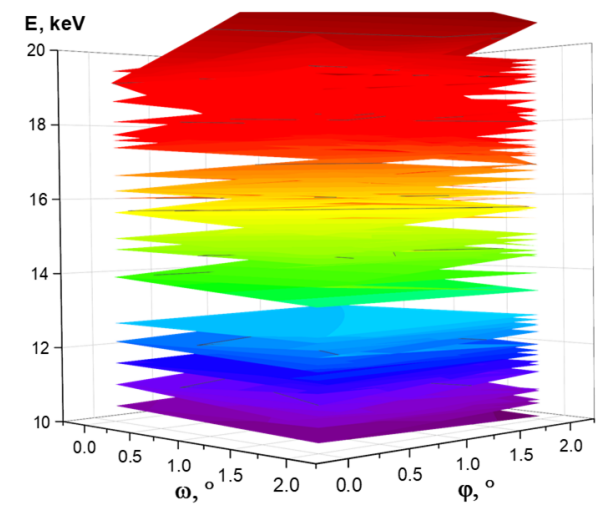

(b)

Figure 4. The simulated glitches in a diamond crystal, (a) for different angles $\phi$ and fixed $\omega=0^{\circ}$; (b) for different angles $\omega$ and $\phi$.

One can see from Figure 4a that for some glitch lines, the energy is decreasing with the angle, while for the others it is increasing. The same can be observed if both angles $\omega$ and $\phi$ are changing (Figure $4 \mathrm{~b}$ ). Therefore, there is no way to avoid glitches during the energy change even with the simultaneous rotation of the optical element, as a glitch line (or plane for rotating both $\omega$ and $\phi$ ) would be intersected. However, it is still possible to measure in 
the following way: locate a rotation with fewer glitches, measure in the "glitch-free" region of energies, then stop the measurements, rotate the optics, and continue the measurements in the next "glitch-free" energy range.

\subsection{Finding the Exact Rotation Axis}

In order to perform precise and controllable rotations, it is necessary to find the exact rotational axis of the crystalline lattice with respect to the beam. So far, only rotations around the Cartesian axes $\mathrm{x}, \mathrm{y}$, and $\mathrm{z}$ have been considered, in addition to assuming that the beam was exactly parallel to one of the axes and that the rotational axes were also aligned with the Cartesian coordinates. In fact, this is usually not the case. In Figure 5a, three glitch spectra of the same sample at different rotation angles $\chi$ (see Figure 1) are presented. If the rotation axis was aligned with the beam, the three spectra would have been overlapped. Due to the small misalignment of the rotational axis with respect to the beam direction, there are some differences in the spectra (Figure 5a). We used each glitch spectrum to determine the exact direction of the incident beam for each measurement. Moreover, using the calculated vectors for each angle $\chi$ we can in fact determine the true rotational axis with high precision. The math behind this calculation is as follows.

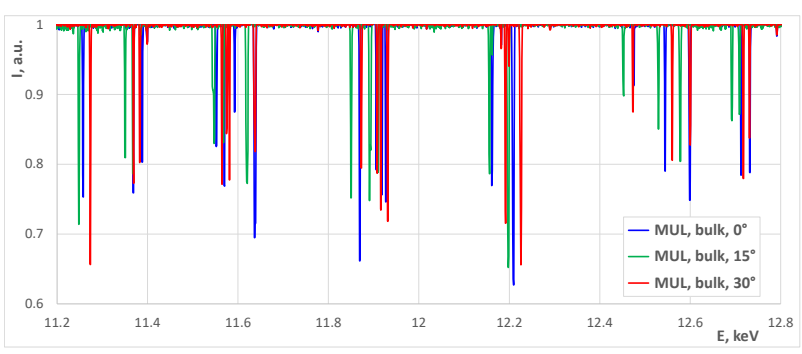

(a)

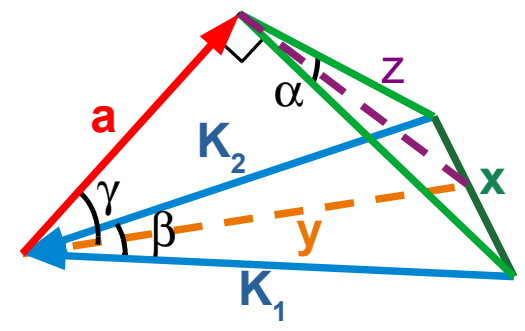

(b)

Figure 5. (a) Three measured glitch spectra at $\chi=0^{\circ}, \chi=15^{\circ}$ and $\chi=30^{\circ}$; (b) schematics of the determination of the rotation axis $a$ from two known vectors $K_{1}$ and $K_{2}$.

Let us assume the vector $K_{2}$ is obtained by the rotation of the vector $K_{1}$ around the axis $a$ by the angle $\alpha$ (Figure $5 b$ ). In our case, we know the vectors $K_{1}$ and $K_{2}$ and the angle $\alpha$, but we need to find the rotation axis $a$. It is obvious that the vector $a$ can be found by rotating vector $y$ around vector $x$ by the angle $\gamma$. Vectors $x$ and $y$ can be easily determined:

$$
\begin{gathered}
y=\left(K_{1}+K_{2}\right) / 2 \\
x=K_{1}-K_{2}
\end{gathered}
$$

To determine angle $\gamma$ we need to find $x, y, z$ :

$$
\begin{gathered}
x=2 K \sin \left(\frac{\beta}{2}\right) \\
y=K \cos \left(\frac{\beta}{2}\right) \\
z=\frac{x}{2 \tan (\alpha / 2)}=\frac{K \sin (\beta / 2)}{\tan (\alpha / 2)}
\end{gathered}
$$

where $K=K_{1}=K_{2}$ is the length of the two vectors.

Thus, the angle $\gamma$ is:

$$
\gamma=\arcsin \left(\frac{z}{y}\right)=\arcsin \left(\frac{\tan (\beta / 2)}{\tan (\alpha / 2)}\right)
$$

The actual rotation of the vector $y$ around the vector $x$ is acomplished using the standard equations for rotating a vector by an angle around an arbitrary axis. 


\section{Results}

\subsection{Determination of the Rotation Axis from Experimental Data}

In our experiment, we measured three spectra (Figure 5a) of the same sample (MUL, bulk part) at three different angles $\chi$ (see Figure 1$): 0^{\circ}, 15^{\circ}$, and $30^{\circ}$. We used these spectra to test the idea of finding the actual rotation axis $\chi$ of the goniometer.

After processing the three spectra we obtained the following values for the direction of the incident vector $K_{0}$ with respect to the cristalline lattice:

$\chi=0^{\circ}:(0.995224,0.066330,-0.071614)$;

$\chi=15^{\circ}:(0.995137,0.067263,-0.071961)$;

$\chi=30^{\circ}:(0.995062,0.068237,-0.072075)$.

Using the proposed equations from the first two measurements (at $\chi=0^{\circ}$ and $\chi=15^{\circ}$ ) we have determined the rotation axis $a$ : $(0.995380,0.068491,-0.067282)$. Using this axis and rotating the $K_{0}$ of the measurement at $\chi=0^{\circ}$ by $30^{\circ}$ we obtained the following calculated value for $K_{0}$ at $\chi=30^{\circ}$ (compared to the measured value):

$(0.995022,0.068780,-0.072104)$-calculated.

(0.995062, 0.068237, -0.072075)-measured.

Hence, we demonstrated that we can easily determine any rotation axis just from two glitch spectra measured at two different rotation angles of that axis.

\subsection{Practical Guide for Measuring without Glitches}

As described earlier, it is absolutely feasible to avoid glitches during the measurements. In Figure 6 the schematics demonstrating the idea of glitch avoidance are presented.

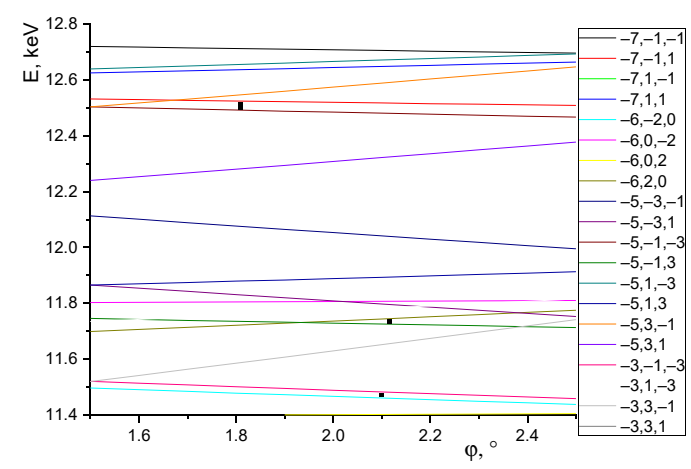

(a)

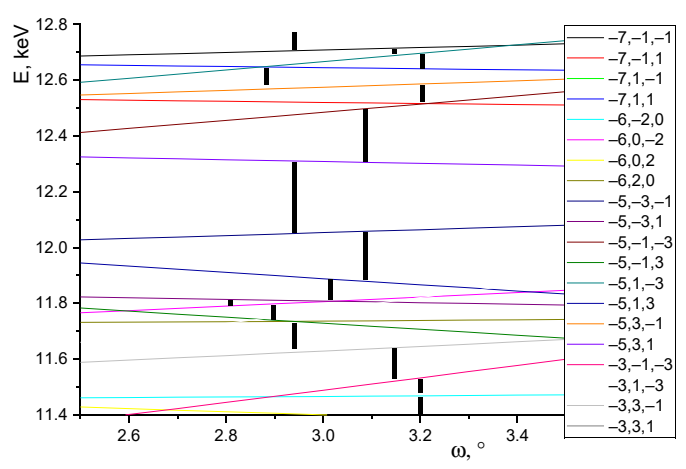

(b)

Figure 6. A possible path (bold black line) for avoiding glitches for a diamond crystal at $11.4-12.8 \mathrm{keV}$. (a) Glitches for fixed $\omega=3^{\circ}$; (b) glitches for fixed $\phi=2^{\circ}$.

The path presented in Figure 6 is a schematic because the two plots are only correct for the fixed value of the other angle $\left(\omega=3^{\circ}\right.$ and $\left.\phi=2^{\circ}\right)$. The true path should be plotted in the $3 \mathrm{D}$ space of $\mathrm{E}, \omega, \phi$-as in Figure $4 \mathrm{~b}$. However, as one can see the rotation of $0.2^{\circ}$ was more than sufficient to avoid glitches (in fact the rotation can be even smaller). In the next chapter, we will discuss whether such a rotation was actually possible.

\section{Discussion}

Let us check how much we can rotate the lens before it gets misaligned. In Figure 7 , detailed schematics of the 1D lenses described in the experimental section (MUL and NDT) are presented.

The diameter of the beam incident on the lens was about $0.1 \mathrm{~mm}$. Considering that it was well-centered in the beginning, the maximum angle the lens can be rotated by, before it becomes misaligned, can be easily calculated. Considering that the minimum radius of curvature of the lenses is $0.2 \mathrm{~mm}$, we allowed the beam to shift by half of this magnitude while it propagates through the lens. Hence, for the MUL lens with a total length of $3 \mathrm{~mm}$ 
and thickness of $0.3 \mathrm{~mm}$, the maximum $\omega$ and $\phi$ angles are $1.9^{\circ}$. For the NDT lens with the length of $8.4 \mathrm{~mm}$ and the thickness of $1 \mathrm{~mm}$ the maximum $\omega$ angle is $0.7^{\circ}$ and the maximum $\phi$ angle is $3^{\circ}$. Therefore, the rotations of $0.2^{\circ}$ demonstrated in Figure 7 can be easily achieved for both lenses.

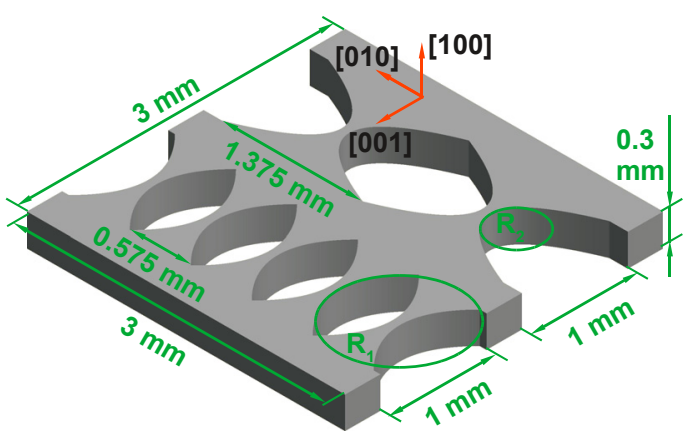

(a)

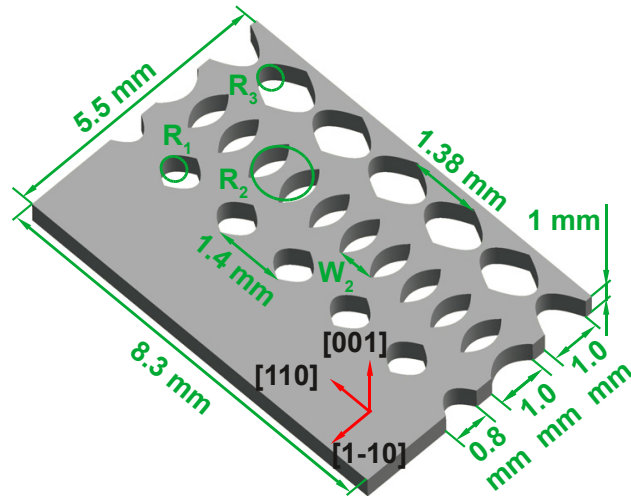

(b)

Figure 7. Schematic of the $1 \mathrm{D}$ lenses used in the experiment. (a) MUL [18]: $\mathrm{R}_{1}=0.2 \mathrm{~mm}, \mathrm{R}_{2}=0.5 \mathrm{~mm}$; (b) NDT [20]: $\mathrm{R}_{1}=0.2 \mathrm{~mm}, \mathrm{R}_{2}=0.5 \mathrm{~mm}, \mathrm{~W}_{2}=0.75 \mathrm{~mm}, \mathrm{R}_{3}=0.2 \mathrm{~mm}$.

In principle, only one rotation axis is enough to avoid most of the glitches. The exceptions are the glitches originating from RLPs with a reciprocal vector that is parallel (or almost parallel) to the rotation axis. Therefore, it is better to have the possibility of rotating the optical element around two axes. For the CRLs, it is usually not a problem because the alignment of the lens has to be performed anyway.

To implement the proposed energy scan, the actual method of scanning has to be changed. The continuous scanning of energy cannot be used-as described already, the scan has to be stopped when it approaches a glitch, then the optical element has to be rotated around one of the axes by a small pre-calculated angle before the scan can be resumed. This method of energy scanning is certainly more time-consuming than the standard one. The actual time of such a modified scan depends on many experimental parameters-for example, the required acquisition time per point for the current sample. However, if the possibility of such a scan is planned during the beamline design stage, the speed of such a scan can be greatly improved.

Quite often, spectroscopy measurements do not require a large energy range. Therefore, the described procedure can be used to find an orientation of the transmissive optical element that makes the small required energy range "glitch-free". An example of the program that can calculate the orientation of a lens for a glitch-free measurement in a small energy range can be found on GitHub [25].

Summarizing everything described in this paper, the algorithm for measuring in the "glitch-free" mode is as follows:

1. Align the crystalline optical element;

2. Measure a small glitch spectrum (0.5-2 keV);

3. Rotate the optical element around one axis by a small amount $\left(0.2^{\circ}\right.$ is enough);

4. Measure a glitch spectrum again;

5. Return the optical element to the aligned orientation and rotate around the second axis (if available), again by $0.2^{\circ}$;

6. Repeat the measurement of the glitch spectrum;

7. Using the three measured spectra find exact rotation axes;

8. Calculate a table of rotations using the available motors for all energies where glitches can be observed;

9. Perform an energy scan stopping at the precalculated energies, changing one of the angles, and resuming the scan. 
It is important to note that even when measuring at some fixed energy, one should check the possibility of "catching a glitch", especially at high X-ray energies, where the density of the glitches in the spectrum is higher. Otherwise, during the tuning of optics, or just due to its heating, one can accidentally satisfy the parasitic diffraction condition causing the intensity of the beam to drop. This would be especially problematic if something is changing during the measurement and the intensity transmitted through the optics is unstable. Therefore, it would be ideal to perform a small energy scan to check if there are no glitches close to the energy at which the measurements would be carried out. If there is a glitch, one can carry out the proper characterization, as described in this paper, or simply rotate the optical element a little and check again (with a small energy scan) if the glitch has disappeared.

\section{Conclusions}

X-ray optics made of single-crystal material have outstanding properties in terms of low background (due to its purity), robustness (especially diamond), and reproducibility. The biggest issue of such optics are the diffraction losses (glitches) observed at some energies due to undesired Bragg diffraction.

Glitches were mostly observed in monochromators due to the fact that this is the mostused single-crystal X-ray optical element. In our following papers, we will present the theory of such glitches and describe methods for avoiding the glitches in monochromators. However, such glitches have a rather limited influence on the diffracted beam due to the typically low penetration depth of X-rays inside the monochromator crystals. On the other hand, glitches in transmissive X-ray optics can be very strong and the transmitted intensity might drop almost to zero.

In this paper, we proposed a method for measuring without glitches. This method is based on the fact that the energy of the glitches is very sensitive to the orientation of the crystalline lattice. Hence, we proposed that an energy scan be carried out in the following way: first, measuring a small spectrum $(0.5-2 \mathrm{keV}$, depending on the energy); then, finding the exact orientation of the optical element and its cell parameters using the procedure described in [7] and programs deposited at [25]; then, calculating small rotations of the optical element close to the energies of the expected glitches and run the energy scan-not continuously, but stopping at precalculated energies, changing the angle according to the precalculated value, and then resuming the scan.

This method requires precise knowledge of the rotational axis of the optical element. We have presented a method to determine each axis using two glitch spectra measured at two different rotations of each axis. In addition, the energy scan itself would need modification to incorporate the rotation of the optical element. However, this should not be a problem but would make the scan a bit longer. All the modifications of the scan and the measurements of glitch spectra do not need big changes to be made to the beamline: the possibility of rotating the optical element, such as CRL, should be present in any case for its alignment. To measure the glitch spectra, one or two intensity sensors are needed.

The entire procedure of avoiding the glitches can be easily automated because most of the calculations do not require human interaction. The procedures used for processing the glitches and the determination of the crystal orientation, with respect to the beam, are described in detail in [7] and are implemented in software that can be downloaded from GitHub [25]. The calculation of the actual energy scan is more beamline-specific, but some example scripts for public usage will be deposited as soon as we perform the corresponding experiment.

We hope that our method can be implemented at different beamlines to solve the issue of glitches in the measured spectra. We plan to perform a demonstration of this method at a synchrotron beamline in the near future, but so far, we want the X-ray community to be aware of this issue and the possible solution that we have described here.

Supplementary Materials: The following are available online at https://github.com/XrayViz/ Glitches. The programs written for this study can be found on GitHub [25]. Additional movies 
explaining the glitches formation (simulated using the program XVis [26]) can be also found at [25].

Author Contributions: Idea for the studies: O.Y.; experiment: N.K., I.S. and A.S.; algorithms development N.K. and O.Y.; computer programs writing: N.K. and O.Y.; data processing: N.K. and O.Y.; writing of the manuscript: N.K. and O.Y.; correction of the manuscript: I.S. and A.S. All authors have read and agreed to the published version of the manuscript.

Funding: This research received no external funding.

Data Availability Statement: Experimental data are available upon reasonable request to the authors. The computer programs could be downloaded from GitHub [25] and are distributed under GPL v.3 license.

Acknowledgments: We gratefully thank the experimental team: Qiuyuan Zhang and Helge B. Larsen from the University of Stavanger, Norway, Ragnvald Mathiesen from the Norwegian University of Science and Technology; and Hermann Emerich from the European Synchrotron Radiation Facility (ESRF) for their contributions. O.Y. acknowledges great support from H.N.Chapman.

Conflicts of Interest: The authors declare no conflict of interest.

\section{References}

1. Snigirev, A.; Kohn, V.; Snigireva, I.; Lengeler, B. A compound refractive lens for focusing high-energy X-rays. Nature 1996, 384, 49-51. [CrossRef]

2. Polikarpov, M.; Emerich, H.; Klimova, N.; Snigireva, I.; Savin, V.; Snigirev, A. Spectral X-ray Glitches in Monocrystalline Diamond Refractive Lenses. Phys. Status Solidi B 2018, 255, 1700229. [CrossRef]

3. Nöhammer, B.; Hoszowska, J.; Freund, A.K.; David, C. Diamond planar refractive lenses for third- and fourth-generation X-ray sources. J. Synchrotron Radiat. 2003, 10, 168-171. [CrossRef] [PubMed]

4. Kononenko, T.V.; Ralchenko, V.G.; Ashkinazi, E.E.; Polikarpov, M.; Ershov, P.; Kuznetsov, S.; Yunkin, V.; Snigireva, I.; Konov, V.I. Fabrication of polycrystalline diamond refractive X-ray lens by femtosecond laser processing. Appl. Phys. A 2016, $122,152$. [CrossRef]

5. Fox, O.J.L.; Alianelli, L.; Malik, A.M.; Pape, I.; May, P.W.; Sawhney, K.J.S. Nanofocusing optics for synchrotron radiation made from polycrystalline diamond. Opt. Express 2014, 22, 7657-7668. [CrossRef]

6. Klimova, N.; Yefanov, O.; Snigirev, A. Predicting glitches of intensity in single-crystal diamond CRLs. In AIP Conference Proceedings; AIP Publishing LLC: Melville, NY, USA, 2020; Volume 2299, p. 060016.

7. Klimova, N.; Yefanov, O.; Snigireva, I.; Snigirev, A. Determination of the Exact Orientation of Single-Crystal X-ray Optics from Its Glitch Spectrum and Modeling of Glitches for an Arbitrary Configuration. Crystals 2021, 11, 504. [CrossRef]

8. Van Zuylen, P.; Van Der Hoek, M.J. Some Considerations on Glitches and The Design Of a Double Crystal Monochromator With Bent Crystals. In Soft X-ray Optics and Technology; International Society for Optics and Photonics: Bellingham, WA, USA, 1986; Volume 733, pp. 248-253.

9. Van Der Laan, G.; Thole, B.T. Determination of glitches in soft X-ray monochromator crystals. Nucl. Instrum. Methods Phys. Res. Sect. A Accel. Spectrometers Detect. Assoc. Equip. 1988, 263, 515-521. [CrossRef]

10. Bauchspiess, K.R.; Crozier, E.D. Crystal Glitches of X-ray Monochromators. In EXAFS and Near Edge Structure III, 2nd ed.; Hodgson, K.O., Hedman, B., Hahn, J.E., Eds.; Springer Proceedings in Physics; Springer: Berlin/Heidelberg, Germany, 1984; Volume 2, pp. 514-516.

11. Li, G.; Bridges, F.; Wang, X. Monochromator-induced glitches in EXAFS data II. Test of the model for a pinhole sample. Nucl. Instrum. Methods Phys. Res. Sect. A Accel. Spectrometers Detect. Assoc. Equip. 1994, 340, 420-426. [CrossRef]

12. Tang, Z.; Zheng, L.; Chu, S.; Wu, M.; An, P.; Zhang, L.; Hu, T. Optimal azimuthal orientation for Si(111) double-crystal monochromators to achieve the least amount of glitches in the hard X-ray region. J. Synchrotron Radiat. 2015, 22, 1147-1150. [CrossRef] [PubMed]

13. Monochromator Crystal Glitch Library. Available online: https://www-ssrl.slac.stanford.edu/xas/glitch/glitch.html (accessed on 28 November 2021).

14. Sutter, J.P.; Boada, R.; Bowron, D.T.; Stepanov, S.A.; Díaz-Moreno, S. Rotation of X-ray polarization in the glitches of a silicon crystal monochromator. J. Appl. Crystallogr. 2016, 49, 1209-1222. [CrossRef] [PubMed]

15. Abe, H.; Aquilanti, G.; Boada, R.; Bunker, B.; Glatzel, P.; Nachtegaal, M.; Pascarelli, S. Improving the quality of XAFS data. J. Synchrotron Radiat. 2018, 25, 972-980. [CrossRef] [PubMed]

16. Wallace, S.M.; Alsina, M.A.; Gaillard, J.-F. An algorithm for the automatic deglitching of X-ray absorption spectroscopy data. J. Synchrotron Radiat. 2021, 28, 1178-1183. [CrossRef] [PubMed]

17. Element Six Ltd. Available online: https:/ / www.e6.com/ (accessed on 16 March 2021).

18. Micro Usinage Laser. Available online: http:/ / micro-usinage-laser.com/ (accessed on 16 March 2021).

19. Polikarpov, M.; Snigireva, I.; Morse, J.; Yunkin, V.; Kuznetsov, S.; Snigirev, A. Large-acceptance diamond planar refractive lenses manufactured by laser cutting. J. Synchrotron Radiat. 2015, 22, 23-28. [CrossRef] 
20. New Diamond Technology. Available online: http://ndtcompany.com/ (accessed on 16 March 2021).

21. Terentyev, S.; Blank, V.; Polyakov, S.; Zholudev, S.; Snigirev, A.; Polikarpov, M.; Kolodziej, T.; Qian, J.; Zhou, H.; Shvyd'Ko, Y. Parabolic single-crystal diamond lenses for coherent X-ray imaging. Appl. Phys. Lett. 2015, 107, 111108. [CrossRef]

22. Zhang, Q.; Polikarpov, M.; Klimova, N.; Larsen, H.B.; Mathiesen, R.; Emerich, H.; Thorkildsen, G.; Snigireva, I.; Snigirev, A. Investigation of glitches induced by single-crystal diamond compound refractive lenses based on crystal orientation. In AIP Conference Proceedings; AIP Publishing LLC: Melville, NY, USA, 2019; Volume 2054, p. 060007.

23. Zhang, Q.; Polikarpov, M.; Klimova, N.; Larsen, H.B.; Mathiesen, R.; Emerich, H.; Thorkildsen, G.; Snigireva, I.; Snigirev, A. Investigation of 'glitches' in the energy spectrum induced by single-crystal diamond compound X-ray refractive lenses. J. Synchrotron Radiat. 2019, 26, 109-118. [CrossRef] [PubMed]

24. Klimova, N.; Snigireva, I.; Snigirev, A.; Yefanov, O. Using diffraction losses in the single-crystal X-ray optics for precise determination of its cell parameters and tuning the monochromator. J. Synchrotron Radiat. 2021. submitted.

25. Programs to Calculate Glitches in Single Crystal X-ray Optics. Available online: https://github.com/XrayViz/Glitches (accessed on 15 March 2021).

26. Yefanov, O.; Kladko, V.; Slobodyan, M.; Polischuk, Y. XVis: An educational open-source program for demonstration of reciprocalspace construction and diffraction principles. J. Appl. Cryst. 2008, 41, 647-652. [CrossRef] 